\title{
Morris, Douglas G. (2020): Legal Sabotage. Ernst Fraenkel in Hitler's Germany
}

Cambridge: Cambridge University Press. 285 Seiten. $£ 85.00$

\author{
Rainer Eisfeld
}

Angenommen: 22. Dezember 2020 / Online publiziert: 7. Januar 2021

(C) Der/die Autor(en) 2021

Diese Studie des New Yorker Strafverteidigers und Rechtshistorikers Douglas Morris, Träger des nach dem ersten afroamerikanischen Richter am Supreme Court benannten Thurgood Marshall Award, wäre kaum entstanden, gäbe es nicht Ernst Fraenkels klassische Darstellung von 1941 über die Koexistenz von reduziertem Normen- und expansivem Maßnahmenstaat im NS-Regime. Betitelt „The Dual State, war sie Ergebnis jener ,gespaltenen“ Existenz Fraenkels, die Morris in eindringlichen Sätzen beschreibt: Legal als Anwalt, der politisch Bedrängten zu helfen suchte (S. 43-93); illegal als Widerständler, der heimlich kursierende regimekritische Streitschriften verfasste (S. 105-123); als Wissenschaftler schließlich (S. 124-146), der den „Doppelstaat“ konzipierte und für den dessen Analyse - so Morris - ,keinen Rückzugsort“ bedeutete, sondern „Fortsetzung der Opposition“ mit anderen Mitteln (S. 145).

Der mehrfach aufgelegte Band bleibt Fraenkels berühmtestes Werk. In meiner Kritik an Fraenkels neopluralistischem Nachkriegsansatz habe ich vor einem halben Jahrhundert die ,klaren analytischen Kategorien des Dual State von 1941“ gerühmt und auf Fraenkels seinerzeitige Distanzierung von Rudolf Smends - Carl Schmitt vorwegnehmenden - Politikbegriff verwiesen (Pluralismus zwischen Liberalismus und Sozialismus, Stuttgart 1972, S. 162-163). Dass ich damals nicht ausführlicher auf den „Doppelstaat“ eingegangen bin, habe ich immer wieder bedauert. Zwei Jahre später erschien die durch Alexander von Brünneck angeregte Rückübersetzung von Manuela Schöps. Ernst Fraenkels Vorwort deutete an, welche Bedingungen das aus Deutschland herausgeschmuggelte Manuskript geprägt hatten, ehe er und seine Frau ins amerikanische Exil flohen (Der Doppelstaat, Frankfurt/Köln 1974, S. 13): „In der Atmosphäre der Rechtlosigkeit und des Terrors“ sei die Urfassung des Buchs

R. Eisfeld ( $\square)$

FB 1, Universität Osnabrück, Osnabrück, Deutschland

E-Mail: rainer.eisfeld@uos.de 
,aus dem Bedürfnis entstanden, diese Erlebnisse und Erfahrungen theoretisch zu erfassen, um mit ihnen innerlich fertig zu werden.“

Andeutungsweise kam Fraenkel (Der Doppelstaat, S. 16) auch auf seine illegale Arbeit im Internationalen Sozialistischen Kampfbund (ISK) zu sprechen: „Wiederholt habe ich im Anschluss an [unsere] Aussprachen deren Ergebnisse in Form von kurzen Aufsätzen [dem ISK-,Inlandsleiter'] Fritz Eberhardt in das Stenogramm diktiert. Sie waren für die Publikation der in Paris erscheinenden Zeitschrift des ISK Sozialistische Warte bestimmt und sind anschließend als illegale Flugblätter in Deutschland verbreitet worden."

Anders als Franz Neumann, mit dem er seit 1927 eine gemeinsame Anwaltspraxis betrieb und der im Mai 1933 nach England floh, versuchte Fraenkel, im NS-Staat weiterzuarbeiten. Infolge seiner Teilnahme am Ersten Weltkrieg blieb er auch nach 1933 zunächst zur Anwaltschaft zugelassen - freilich nicht, ohne auf Schritt und Tritt Diskriminierungen und Schikanen zu erfahren: Auf bedrückende Weise skizziert Morris (S. 10-38) die schrittweise Durchbrechung des Normenstaats beim Umgang des Regimes mit jüdischen Anwälten.

Was befähigte Fraenkel, diesen Anforderungen bis zur drohenden Verhaftung und Flucht 1938 standzuhalten? Morris' Antwort lautet (S. 199-210): Er trotzte der Furcht vor dem Tod, weil er von der Notwendigkeit zum Handeln durchdrungen war; und er trotzte der distanzlosen Identifizierung mit seinem Beruf, weil er sich vorrangig als Sozialist verstand. Aufrechterhaltene politische Kontakte verhinderten geistige und emotionale Verarmung. Dass er vor dem Volksgerichtshof nicht plädieren durfte, schützte ihn vor übergroßer behördlicher Aufmerksamkeit; seine Praxis hatte er ohnehin in seine Wohnung verlegt (S. 206-207). Und Fraenkel blieb, Morris' Urteil zufolge, in seinem Doppelleben ,,vorsichtiger Realist“, der im Doppelstaat zu „manövrieren“ lernte, seinem Gewissen ebenso verpflichtet wie intellektueller und emotionaler „Selbstdisziplin“ (S. 104, 203-204, 210).

Morris porträtiert nicht nur einfühlsam die Persönlichkeit des 35- bis 40-jährigen Fraenkel. Er leuchtet auch sorgfältig dessen unmittelbares Umfeld im Widerstand aus, vornehmlich durch Kontrastierung (S. 152-187) mit den differierenden ideellen Prämissen und Handlungsweisen Hermann Brills (1938 verhaftet, Mitautor des „,Buchenwalder Manifests“) und Martin Gaugers (1941 ermordet; wer Illusionen über die katholische Kirche im NS-Staat hegt, sollte dazu S. 172 lesen). Und er arbeitet mit großer Prägnanz die unterschiedlichen Interpretationsstränge heraus, denen die „intellektuellen Zwillinge“ (S. 174) Fraenkel und Neumann folgten, als sie - der eine vor Ort, der andere aus dem Exil - den Charakter des NS-Regimes zu erklären suchten.

Neumann wie Fraenkel stimmten darin überein, dass letztendlich für den Nationalsozialismus „Politik an Stelle des Rechts trat“" (S. 179). Neumann nannte dies, den ursprünglich auf Schmitt gemünzten Begriff aufgreifend, Dezisionismus - ,die Ersetzung des rationalen Gesetzes durch den politischen Befehl“" (Behemoth, Köln/ Frankfurt 1977, S. 522). Fraenkel bezeichnete es, inhaltlich identisch, als Stellung der ,gesamte[n] Rechtsordnung [...] zur Disposition der politischen Instanzen“ (Der Doppelstaat, S. 88). An diesem Punkt jedoch trennten sich, wie Morris darlegt (S. 178-179), beider Wege. Fraenkel suchte als analytischen Ausgangspunkt einen Rest rationaler Naturrechtsvorstellungen zu retten. Er betonte, in den Worten von 
Morris, die Dominanz des fortbestehenden - wenn auch als Normenstaat immer weiter reduzierten - Rechts durch die Politik. Neumann hingegen argumentierte auf der Grundlage eines Rechtsbegriffs, der identisch war mit dem während der liberalen Epoche durchgesetzten abstrakt-allgemeinen Charakter des - parlamentarisch verabschiedeten - Gesetzes, verbunden mit richterlicher Unabhängigkeit. Für ihn galt folglich, so Morris, dass im NS-Staat die Politik das Recht ersetzt hatte. Was übrig blieb, waren ,technische Regeln“. Fraenkel dagegen, dessen pragmatische Züge Morris wiederholt betont, sah wenig Sinn darin, ,die Existenz von irgendeiner Art Gesetz zu bestreiten“, wenn er bei Gerichten plädierte, „die in irgendeiner Art Rechtssystem funktionierten“ (S. 179).

Morris' umfänglich recherchierte Studie (,,in einem Archiv zu sitzen, ist für mich so aufregend wie für andere die Besichtigung einer antiken Stadt"“, S. XIII) wurde, siehe Vorwort, engagiert unterstützt von Hubertus Buchstein und Volker Berghahn (Columbia University). Sie ergänzt vorzüglich die Materialien in dem durch Brünneck 1999 edierten Band 2 der Gesammelten Schriften Fraenkels (Nationalsozialismus und Widerstand). Morris hat ein herausragendes, ein berührendes Buch vorgelegt. Das Titelbild unterstreicht die Besonderheit des Werks. Es gibt eine Zeichnung des Buchenwald-Häftlings Herbert Sandberg (1908-1991) wieder, und es entstammt dem Archiv der Stiftung Gedenkstätten Buchenwald und MittelbauDora. Deren Kuratorium habe ich zwei Jahrzehnte angehört. Auch so schließt sich ein Kreis.

Funding Open Access funding enabled and organized by Projekt DEAL.

Open Access Dieser Artikel wird unter der Creative Commons Namensnennung 4.0 International Lizenz veröffentlicht, welche die Nutzung, Vervielfältigung, Bearbeitung, Verbreitung und Wiedergabe in jeglichem Medium und Format erlaubt, sofern Sie den/die ursprünglichen Autor(en) und die Quelle ordnungsgemäß nennen, einen Link zur Creative Commons Lizenz beifügen und angeben, ob Änderungen vorgenommen wurden.

Die in diesem Artikel enthaltenen Bilder und sonstiges Drittmaterial unterliegen ebenfalls der genannten Creative Commons Lizenz, sofern sich aus der Abbildungslegende nichts anderes ergibt. Sofern das betreffende Material nicht unter der genannten Creative Commons Lizenz steht und die betreffende Handlung nicht nach gesetzlichen Vorschriften erlaubt ist, ist für die oben aufgeführten Weiterverwendungen des Materials die Einwilligung des jeweiligen Rechteinhabers einzuholen.

Weitere Details zur Lizenz entnehmen Sie bitte der Lizenzinformation auf http://creativecommons.org/ licenses/by/4.0/deed.de. 